\title{
Mesoporous silica templated-albumin nanoparticles with high doxorubicin payload for drug delivery assessed with a 3-D tumor cell model
}

Mathilde Ménarda,b, Florent Meyer*,b, Ksenia Parkhomenkoc ${ }^{c}$ Cédric Leuvreya ${ }^{a}$ Grégory Francius ${ }^{d}$, Sylvie Bégin-Colin*,a, Damien Mertz*a

anstitut de Physique et Chimie des Matériaux de Strasbourg (IPCMS), UMR 7504, CNRS, Université de Strasbourg, 23, rue du Loess, BP 43, 67034 Strasbourg, France. E-mails: damien.mertz@ipcms.unistra.fr sylvie.begin@ipcms.unistra.fr

bUniversité de Strasbourg, INSERM, UMR_S 1121 Biomatériaux et bioingénierie, FMTS, 11 rue Humann, 67085 Strasbourg, Cedex, France. E-mail : fmeyer@unistra.fr

'Institut de Chimie et Procédés pour l'Energie l'Environnement et la Santé, 25 rue Becquerel 67087 Strasbourg

${ }^{d}$ CNRS - Université de Lorraine, Laboratoire de Chimie Physique et Microbiologie pour l'Environnement, LCPME, UMR 7564, Villers-lès-Nancy, F-54600, France.

\begin{abstract}
.
Human serum albumin (HSA) nanoparticles emerge as promising carriers for drug delivery. Among challenges, one important issue is the design of HSA nanoparticles with a low mean size of ca. $50 \mathrm{~nm}$ and having a high drug payload. The original strategy developed here is to use sacrificial mesoporous nanosilica templates having a diameter close to $30 \mathrm{~nm}$ to drive the protein nanocapsule formation. This new approach ensures first an efficient high drug loading (ca. 30\%) of Doxorubicin (DOX) in the porous silica by functionalizing silica with an aminosiloxane layer and then allows the one-step adsorption and the physical cross-linking of HSA by modifying the silica surface with isobutyramide (IBAM) groups. After silica template removal, homogenous DOX-loaded HSA nanocapsules (30-60 nm size) with high drug loading capacity (ca. $88 \%$ ) are thus formed. Such nanocapsules are shown efficient in multicellular tumor spheroid models (MCTS) of human hepatocarcinoma cells by their significant growth inhibition with respect to controls. Such a new synthesis approach paves the way toward new protein based nanocarriers for drug delivery.
\end{abstract}

\section{Introduction}

Designing nanoparticles as controlled drug delivery systems for cancer therapy has attracted great attention to overcome the problems of chemotherapeutics side effects such as the wellknown cardiotoxicity of anthracyclines.[1-5] Human serum albumin (HSA) derived nanoparticles show high potential for drug delivery.[6,7] HSA, the most abundant plasma protein $(35-50 \mathrm{~g} / \mathrm{L}$ human serum), has biocompatible, non-immunogenic and biodegradable properties.[8] 
Furthermore, HSA shows high average half-life of 19 days which enhances protein tumor uptake.[9]

HSA nanoparticles can be prepared through a multitude of techniques, the main one being desolvation,[10-12] in which water soluble drugs are loaded either by incubation in the formed nanoparticles, or by incorporation in the albumin solution before particle formation and crosslinking.[13] For example Dreis et al.[14] prepared HSA nanoparticles and investigated the loading of the anti-cancer drug doxorubicin (Dox), by incubation (adsorption on particle surface) compared to incorporation. For both loading strategies, high loading efficiencies (70-95 wt\%) were obtained and Dox loaded HSA nanoparticles showed improved anti-cancer effects on in-vitro cell cultures compared to free Dox molecules. However, one of the main important drawback generally encountered in such synthesis strategies is the use of toxic cross-linkers such as glutaraldehyde or formaldehyde. The potential release of such cross-linkers can induce some toxicity as glutaraldehyde is known to be genotoxic, mutagenic and carcinogenic.[15-17] Furthermore, there is a need to design drug delivery systems with mean sizes below 100 and even $50 \mathrm{~nm}$ to ensure their biodistribution and accumulation in diseases body parts.[18,19]

Considering the promising properties of HSA nanoparticles as drug delivery systems and to face the main challenges of high drug loading/delivery and mean sizes suitable to envision in vivo applications, an original strategy based of the use of silica templates has been innovatively adapted. Indeed, we and co-workers previously developed an approach to form tight and robust HSA micron and sub-micron sized particles assembled non-covalently via isobutyramide (IBAM) grafts and without the need of further cross-linking or addition of adjuvants.[20-22] In this strategy, a sacrificial template of silica is first modified with amine groups on which isobutyrylchloride is grafted to form IBAM-functionalized surface. Then, HSA is coated on IBAMmodified silica by simple adsorption in aqueous solution. The driving force to immobilize HSA to IBAM groups was shown to be of a non-covalent nature and was assumed to result from strong hydrogen-bonding interactions.[20,21] Silica nanoparticles, which were extensively studied in the past two decades, appear as particularly relevant and original sacrificial templates. Indeed, they own outstanding intrinsic features like simple and well-referred synthesis processes leading to nanoparticles with controlled size and shape, tunable morphologies, tunable pore structure and easy surface modification.[23-25] Furthermore using plain or mesoporous silica nanoparticles influences the design of the final HSA nanoparticles obtained after complete dissolution of silica template with hydrofluoric acid (HF): full silica particles lead to the formation of HSA hollow capsules [21] whereas mesoporous silica nanoparticles with large porosities, allowing the diffusion of HSA into the pores, will form replicate HSA nanoparticles.[22] However the decrease of the mean size of such protein and polymer capsules, in order to reach the sub-micron scale is currently a great challenge, and to the best of our knowledge the use of template below $100 \mathrm{~nm}$ is scarcely investigated.[26,27]

Considering the specifications of drug delivery systems with a high drug loading, mesoporous silica nanoparticles (MSNs) are well-known drug delivery systems since Vallet-Regi et al. reported in 2001 the loading of drug molecules within MCM-41 materials.[28] Indeed, MSNs exhibit large 
surface area and pore volume providing great potential for drug adsorption and loading within the pore channels. In addition, the feature of tunable porosities enables better control of the drug loading and the type of drug loaded. Indeed, MSNs with large porosities ( $>3 \mathrm{~nm}$ ) can load large drug molecules, siRNA, plasmid, proteins, quantum dots, nanoparticles[29] whereas MSNs with small porosities $(\sim 3 \mathrm{~nm})$ are well known for the loading of small drug molecules such as anthracyclines, ibuprofen, cisplatin, aspirin etc[30,31] Therefore, besides their use as sacrificial templates for the formation of self-supported macromolecule assemblies, MSNs can serve in a first place as nanocontainers for drug loading and drive after their removal the final shape of the protein-based particle.

Herein, we report the design of MSNs templated self-supported HSA nanocapsules (size below $50 \mathrm{~nm}$ ) with a high Dox content and demonstrate their potential as drug delivery system using a 3D tumor cell model. The MSNs addressed for this study exhibited a raspberry (RB) morphology with a narrow size distribution centered at about $30 \mathrm{~nm}$ and small porosities of $3 \mathrm{~nm}$.[32] They are thus called RB NPs. This porosity has the benefits on one hand to be large enough to load chemotherapeutics into it by impregnation but, on another hand, to be small enough to provide an efficient HSA coating at the surface of the RB NPs leading thus to hollow-capsules formation after silica removal. As illustrated in Scheme 1, RB NPs were first synthesized and their surface functionalized with amine groups by using aminopropyltriethoxysilane (APTS) prior Dox loading. Indeed, very recently, we showed that an aminosilane layer condensed at very high silane concentration at the surface of carbon nanotubes@MS composites allowed to reach very high payloads of Dox ( $\geq 50 \%$ ) depending on the feed weight ratio (fwr).[33] After such drug loading, the amino functions from APTS were modified with isobutyrylchloride to form IBAM moieties. Then, HSA was absorbed on the IBAM-functionalized surface forming a tight protein shell onto the RB NPs through assumed noncovalent H-bonding with IBAM. Finally, after removal of mesoporous silica sacrificial templates dissolved in $\mathrm{HF} / \mathrm{NH}_{4} \mathrm{~F}$ buffer ( $\mathrm{pH} \sim 5$ ) and dialysis to remove fluoride ions, self-supported Dox loaded HSA nanocapsules were formed. To assess their efficiency as drug delivery systems, cytotoxicity and growth inhibition studies were carried out on human hepatocarcinoma spheroids.

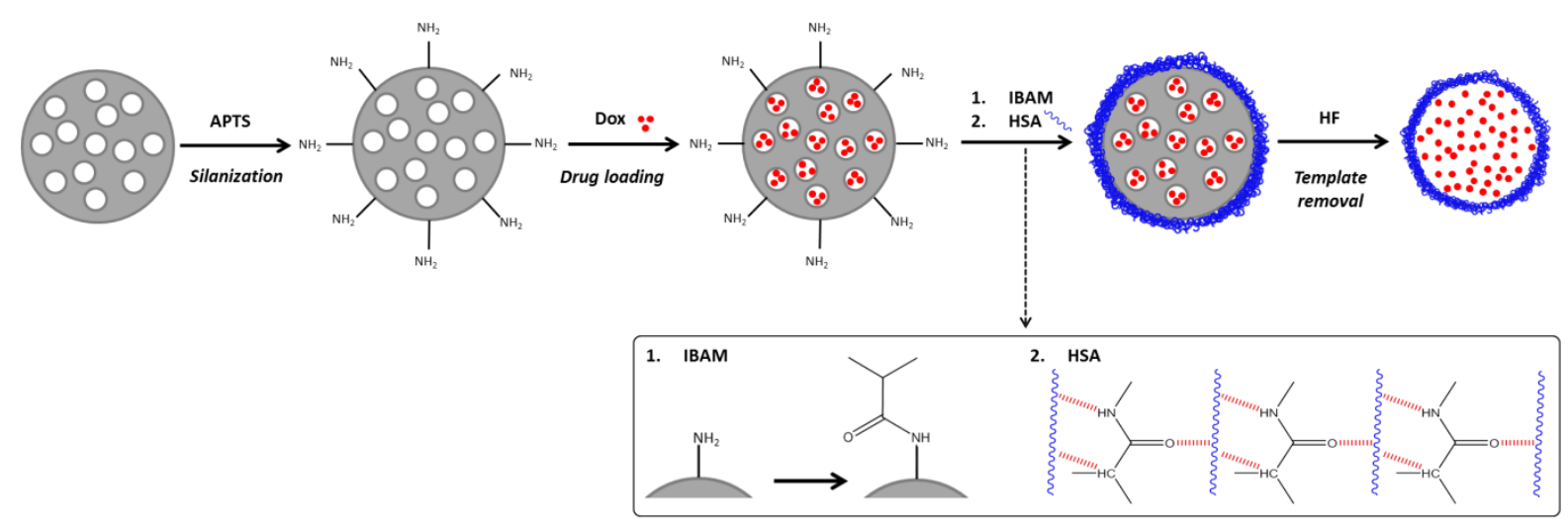

Scheme 1. Schematic representation of the Dox loaded HSA nanocapsules synthesis strategy. 


\section{Experimental}

\section{A. Materials}

Tetraethyl orthosilicate (TEOS, Sigma-Aldrich), ammonium hydroxide solution $\left(\mathrm{NH}_{3 a q .25 \%}\right)$, triethanolamine $\left(\mathrm{TEAH}_{3}\right.$, Sigma-Aldrich), cetyltrimethyl-ammonium bromide (CTAB, Roth), Human Serum Albumin (HSA, Sigma-Aldrich), isobutyrylchloride (IBC, Sigma-Aldrich), Doxorubicin hydrochloride (Dox, 99\%, OChem Inc, DesPlaines, IL), N,N-dimethylformamide (DMF, Sigma-Aldrich), fluorescein isothiocyanate (FITC), 3-aminopropyl-triethoxysilane (APTES, Sigma-Aldrich) and with poly(2hydroxyethyl methacrylate) (poly-HEMA, Sigma-Aldrich) were used as received.

\section{B. Procedures}

Synthesis of MSNs with a raspberry morphology (RB NPS). The RB NPs were synthesized according to a modified literature method.[32] Briefly, $960 \mathrm{mg}$ of CTAB were mixed with $230 \mathrm{mg}$ of $\mathrm{TEAH}_{3}$ in $63 \mathrm{~mL} \mathrm{of}$ Milli-Q water. This mixture was first placed for $1 \mathrm{~h}$ under sonication then $1 \mathrm{~h}$ at $80^{\circ} \mathrm{C}$ until the components were completely dissolved. Next, $4 \mathrm{~mL}$ of TEOS was quickly added to the above solution under stirring. The mixture was stirred at $80^{\circ} \mathrm{C}$ for $2 \mathrm{~h}$. The as-synthesized RB NPs were filtered, washed and dried in an oven at $100{ }^{\circ} \mathrm{C}$ for $24 \mathrm{~h}$. The resulting powders were then calcined at $550{ }^{\circ} \mathrm{C}$ for $6 \mathrm{~h}$ to burn the surfactant molecules left in the porosities.

Amino-functionalization of $\mathrm{RB}: \mathrm{RB}-\mathrm{NH}_{2} \mathrm{NPS}$. To functionalize the RB NPs with a layer of primary amine groups, the calcined RB NPs were re-dispersed in $40 \mathrm{~mL}$ of EtOH at a concentration of $1.5 \mathrm{mg} \cdot \mathrm{mL}^{-1}$ with $960 \mu \mathrm{L}$ of $\mathrm{NH}_{3}$ and $4 \mathrm{~mL}$ of APTES and the mixture was stirred for 2 hours at room temperature. The amino-functionalized MSNs (RB- $\mathrm{NH}_{2}$ NPs) were then washed three times with EtOH by centrifugation.

Dox loading. Dox loading into $\mathrm{RB}-\mathrm{NH}_{2}$ porosities was performed in Milli-Q water by simple soaking. RB$\mathrm{NH}_{2}$ NPs dispersed at $0.5 \mathrm{mg} \cdot \mathrm{mL}^{-1}$ were mixed with Dox at a feed weight ratio (fwr) of $100 \%$.

$$
\text { fwr } \%=\frac{\text { weight of Dox fed }}{\text { weight of NPs }} \times 100
$$

The mixture was stirred at room temperature in the dark for $24 \mathrm{~h}$. Then the Dox loaded RB-NH $\mathrm{NPs}_{2}$ were collected by centrifugation.

IBAM grafting and HSA coating. Before the HSA adsorption, the $\mathrm{RB}^{-\mathrm{NH}_{2}} \mathrm{NPs}$ were functionalized with IBAM groups. In a typical procedure, $300 \mu \mathrm{L} \mathrm{Et}_{3} \mathrm{~N}$ were mixed with $1.5 \mathrm{~mL}$ of RB- $\mathrm{NH}_{2}$ at $10 \mathrm{mg} \cdot \mathrm{mL}^{-1}$ in $\mathrm{DMF}^{-}$ before the quick addition of IBC: DMF (405 $\mu \mathrm{L}: 3 \mathrm{~mL})$ mixture forming a precipitate with $\mathrm{RB}^{-\mathrm{NH}_{2}} \mathrm{NPs}_{\text {. }}$ After $1 \mathrm{~h}$ of reaction under stirring, $15 \mathrm{~mL}$ of HSA at $2 \mathrm{mg} \cdot \mathrm{mL}^{-1}$ in Milli-Q water were added. The precipitate dissolved immediately after the addition of the aqueous solution and the mixture was put under stirring for additional $2 \mathrm{~h}$. The resulting RB-HSA NPs were collected and washed several time in Milli-Q water by centrifugation. 
Capsules formation by silica dissolution. The RB sacrificial templates were dissolved with the use of ammonium fluoride buffered HF. Basically, $150 \mu \mathrm{L}$ of RB-HSA or RB-Dox-HSA NPs at $2 \mathrm{mg}^{-\mathrm{mL}^{-1} \text { were }}$ mixed with $150 \mu \mathrm{L}$ of ammonium fluoride buffered $\mathrm{HF}$ ( $30 \mathrm{mM}, \mathrm{pH}$ ) for $1 \mathrm{~h}$ before being dialyzed for 3 days against $3 \mathrm{~mL}$ of Milli-Q water (changed every day).

Preparation of Huh7 multicellular tumor spheroid model (MCTS). The Huh7-luc cells were cultured in a Dulbecco's Modified Eagle's Medium (DMEM) extended with $10 \%$ of fetal calf serum (FCS), $5 \%$ of PS and $1 \%$ of non-essential amino acids. Prior to the culture, sterile 96-well plates were coated with poly-HEMA in order to avoid cell adhesion at the well-bottom. The coating was done by addition of a $0.5 \%$ (w/v) polyHEMA solution in ethanol ( $50 \mu \mathrm{L}$ ) into each well of a 96-well plate. For ensuring a total surface coating, the plate was put at $38^{\circ} \mathrm{C}$ for $24 \mathrm{~h}$ until complete ethanol evaporation. The wells were then washed three times with PBS $(100 \mu \mathrm{L})$. Adherent Huh-7 were treated with trypsin and suspended in complete medium at 5.3 $10^{3}$ cell $/ \mathrm{mL}$. The cell suspension $(15 \mu \mathrm{L})$ was deposited as a drop on the inside face of the 96 -well plate lid. The lid was then replaced on the PBS-filled plate and the cells in the hanging drop were left to grow in the incubator for 3 days. During these three days the cells present in the drop sedimented at the medium/air interface, aggregated and developed finally with subsequent proliferation and deposition of ECM into a multicellular tumor spheroid.[34] The PBS was then discarded and replaced with complete cell culture medium $(100 \mu \mathrm{L})$. Each drop containing one single spheroid was then transferred from the lid into the well by centrifugation at $500 \mathrm{RPM}$ for $1 \mathrm{~min}$. MCTS were cultivated further at $37^{\circ} \mathrm{C}$ in $5 \% \mathrm{CO}_{2}$.

Evaluation of antitumoral effect on MCTS. The experiments were carried out in octuplicate in 96-well plate using the spheroids as previously described. NPs were diluted at various concentrations in complete cell culture medium. NPs are immediately added to each spheroid by further dilution into the cell culture medium $(100 \mu \mathrm{L})$. The spheroid growth was examined using a Nikon Eclipse TS100 microscope and a $10 \mathrm{X}$ objective. Images were recorded at different days with a Nikon Color CCD DS-Fi2 Camera and a DS-L3 camera controller. The area (A) of each spheroid was measured using the NIH Image J software and reported in $\mu \mathrm{m}^{2}$. The MCTS growth (G) was calculated relative to the initial area (A0; day 0) and express as relative growth versus control using the following equation:

$$
G=\frac{P_{t}-P_{0}}{P_{0}} \times 100
$$

Where

$$
P_{t}=\frac{A_{t_{X}}}{A_{t_{0}}}
$$

And

$$
P_{C}=\frac{A_{c X}}{A_{c 0}}
$$

with $A_{t}$ the area of treated MCTS, $A_{C}$ the area of control MCTS and X the number of days.

\section{Characterization Methods.}


TEM imaging. The morphology and diameter of the RB MS NPs were determined by transmission electron microscopy (TEM, JEOL 2100) operating at an accelerating voltage of $200 \mathrm{kV}$. The average diameter of the NPs was calculated by measuring on ImageJ software at least 100 NPs from TEM images.

SEM imaging. The size and morphology of RB MS were characterized by scanning electron microscopy (Gemini500-ZEISS- $1 \mathrm{~nm}$ resolution). One drop of the sample, in ethanol or water solution, was deposited on a silicon slide and then the solvent was evaporated.

$\mathrm{N}_{2}$ isotherm adsorption. Nitrogen adsorption isotherms were obtained on a Micrometrics ASAP 2420 Accelerated Surface Area and Porosimetry System at 77K under continuous adsorption conditions. Brunauer-Emmett-Teller (BET) method was used to determine the surface area as well as the total internal pore volume at $\mathrm{P} / \mathrm{P}_{0}=0.97$. By applying the Barrett-Joyner-Halenda $(\mathrm{BJH})$ method, the surface area, the cumulative pore volume, and the mesoporous pore size distribution (PSD) are determined during the adsorption and desorption steps. The microporosity pore size distribution (porosities $<1 \mathrm{~nm}$ ) was calculated with the Horvath-Kawazoe model.

Fourier Transform Infra-Red Spectroscopy (FTIR). The surface composition of the NPs at different steps of synthesis was verified by Fourier Transform Infra-Red Spectroscopy (FTIR) (Perkin Elmer instrument).

Thermogravimetric analysis (TGA). TGA was carried out on a TA SDT 600 instrument in order to determine the Drug loading content (DLC) and the Coating content (CC):

$$
\begin{gathered}
\text { DLC } \%=\frac{\text { weight of Dox loaded }}{\text { weight of NPS }} \times 100 \\
C C \%=\frac{\text { weight of HSA adsorbed }}{\text { weight of } N P S} \times 100
\end{gathered}
$$

Before the analyses, the samples in suspensions were freeze dried. For the runs, the samples were heated from 25 to $1000^{\circ} \mathrm{C}$ at a rate of $5^{\circ} \mathrm{C} / \mathrm{min}$.

AFM imaging in liquid phase. AFM images and force spectroscopy measurements were performed using a Bioscope Resolve instrument (Bruker Nano, Palaiseau, France). Nanoparticles were deposited onto PEI coated silicon wafer during 10 minutes and then extensively rinsed with water prior to morphology and mechanical analyses in liquid phase by AFM. Topographical images of nanoparticles were performed in peakforce tapping ${ }^{\mathrm{TM}}$ mode and mechanical analysis derived from peakforce QNM mode. Silicon nitride cantilevers of conical shape purchased from Bruker (Peakforce HIRS-FA, Bruker, France) with spring constant of about 0.32-0.35 nN/nm were used for both imaging and nanomechanical measurements. All images were recorded with a resolution of $256 \times 256$ pixels and a scan rate of $1 \mathrm{~Hz}$. Nanomechanical properties of nanoparticles under solvent condition were extracted from the analysis of at least 4 peakforce captured (PFC) images at different locations over the silicon wafer surface. The elastic modulus $E$ was calculated by analyzing the force-indentation curves according to the Sneddon model.[35] In this model, the Young modulus is related to the applied force according to the equation given below:

$$
F=\frac{2 E \tan \alpha}{\pi\left(1-v^{2}\right)} \delta^{2} f_{B E C C}
$$


where $\delta$ is the indentation depth, $v$ the Poisson coefficient, $\alpha$ the semi-top angle of the tip and $f_{\mathrm{BECC}}$ is the bottom effect cone correction function that take into account the presence of the substrate stiffness. [36] All the PFC were analyzed with an automatic Matlab algorithm described elsewhere.[37]

\section{Results and discussion}

\section{A. Synthesis and characterization of RB NPs and surface modification with APTS}

The morphology and structure of as-synthesized RB NPs before any functionalization were characterized by TEM and SEM imaging. As depicted in Figure 1.A the synthesis yielded uniform RB NPs of $c a .30 \mathrm{~nm}$ in size with apparent porosities throughout the entire particle. The RB NPs statistical average diameter was measured of $c a .33 \pm 4 \mathrm{~nm}$ and the resulting histogram is presented in Figure 1.B. The size distribution and morphology of the RB NPs were also confirmed by SEM images, which depict very nicely the nanostructure and demonstrated that the pore network was open to outside (Figure 1.C).

The $\mathrm{N}_{2}$ adsorption-desorption isotherms, (Figure S1.A) exhibited according to IUPAC classification, a type IV isotherm which is characteristic for mesoporous channel. The BET surface area and the total pore volume of the calcined RB NPs were measured at ca. $708 \mathrm{~m}^{2} \cdot \mathrm{g}^{-1}$ and $1.62 \mathrm{~cm}^{3} \cdot \mathrm{g}^{-1}$ respectively. These values are consistent to those describe in literature for RB NPs (BET surface area: $675 \mathrm{~m}^{2} \cdot \mathrm{g}^{-1}$; total pore volume: $1.27 \mathrm{~cm}^{3} \cdot \mathrm{g}^{-1}$ [32]). The corresponding pore size distribution (PSD) (Figure S1.B) calculated for pore sizes above $2 \mathrm{~nm}$ using the $\mathrm{BJH}$ method exhibited a single peak centered on $2.8 \mathrm{~nm}$. With the Horvath-Kawazoe method (used for porosities $<1 \mathrm{~nm}$ ) a single peak at $0.7 \mathrm{~nm}$ was recorded. Via those two PSD calculation methods, we found out that these RB NPs showed a bimodal porosity centered at 7 and $28 \AA$.
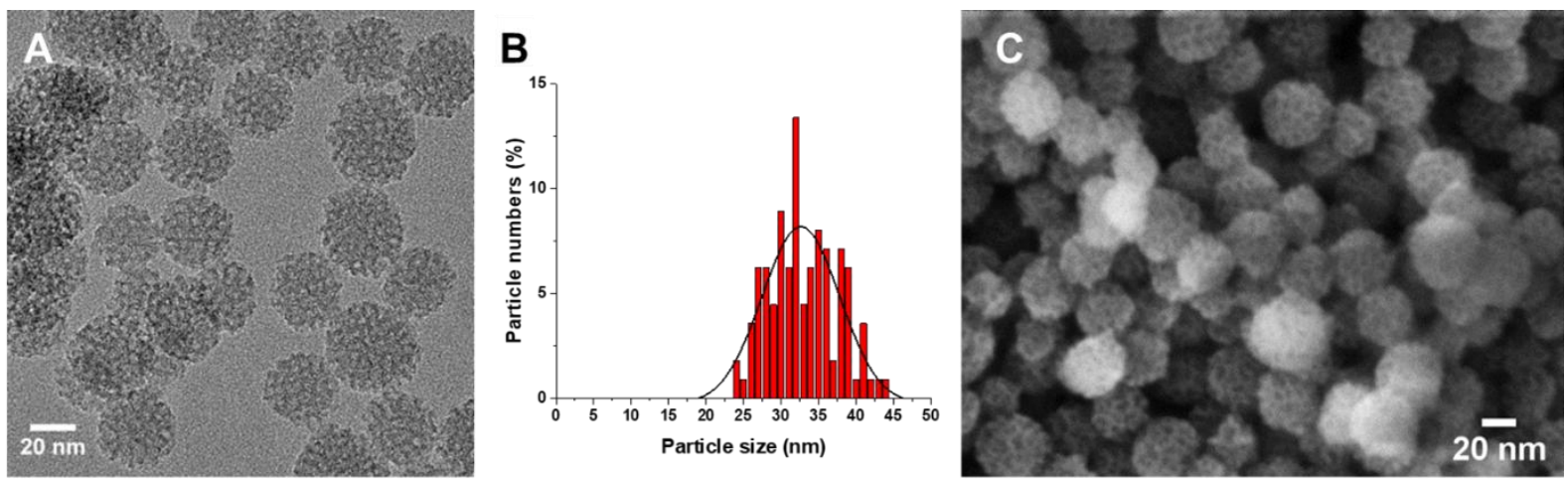

Figure 1. A) TEM image of RB NPs. B) Particle size distribution of RB NPs from TEM pictures $(>100$ particles). C) SEM image of RB NPs.

RB NPs were then functionalized with amine groups with a concentration ensuring the formation an aminosilane layer providing high drug loading.[33] FTIR-ATR analyses were achieved to confirm the RB surface modification by APTS. Figure $\mathbf{S} 2$ compares the IR spectra of RB NPs with $\mathrm{RB}^{-\mathrm{NH}_{2}} \mathrm{NPs}$. The intense and broad absorption peak centered at $1065 \mathrm{~cm}^{-1}$ is assigned to $\mathrm{Si}-\mathrm{O}-\mathrm{Si}$ asymmetric stretching vibration, whereas the peak around $800 \mathrm{~cm}^{-1}$ is assigned to Si-O-Si symmetric stretching vibrations. The peak at 970 
$\mathrm{cm}^{-1}$ is attributed to $\mathrm{Si}-\mathrm{OH}$ bond. Finally, the IR band at $450 \mathrm{~cm}^{-1}$ is due to $\mathrm{O}-\mathrm{Si}-\mathrm{O}$ bending vibrations. The appearance of new peaks for the $\mathrm{RB}-\mathrm{NH}_{2}$ sample at 1356 and $1408 \mathrm{~cm}^{-1}$, specific for the stretching bands of C-N groups, and at 1573 and $1618 \mathrm{~cm}^{-1}$, assigned to the bending vibration of $\mathrm{N}-\mathrm{H}$ groups, proved the effective amino-functionalization of the RB surface.

\section{B. Synthesis and characterization of RB-Dox-HSA NPs}

Then, RB-Dox-HSA and the corresponding Dox free RB-HSA NPs were obtained according to the procedure described in the Experimental section. Dox loading into $\mathrm{RB}-\mathrm{NH}_{2}$ porosities was performed in

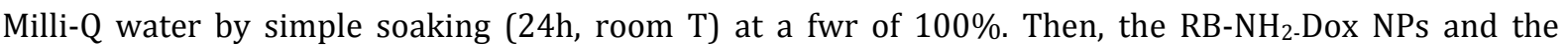
corresponding Dox free RB- $\mathrm{NH}_{2}$ NPs were functionalized with IBAM groups by reaction between amine groups and IBC reagents in the presence of Et3N. After $1 \mathrm{~h}$ of reaction under stirring, HSA was adsorbed at 2 mg.mL ${ }^{-1}$ in Milli-Q water and the resulting RB-DOX-HSA NPs or RB-HSA NPs were washed several times in water. The protein coating and the Dox loading were first characterized by ATR-IR (Figure S3). For both the RB-HSA and RB-Dox-HSA NPs samples in comparison to raw RB NPs, multiple peaks in the ranges of $1300-1800 \mathrm{~cm}^{-1}$ and $2800-3000 \mathrm{~cm}^{-1}$ evidenced the presence of various organic groups usually encountered with polypeptides.

The RB-Dox-HSA NPs were then visualized by TEM (Figure 2.A) and SEM (Figure 2.B) analyses. Both imaging showed RB NPs tangled in a dense HSA network. Thus, the RB NPs seemed to form large aggregates that were trapped into HSA. These particles being collected after centrifugation, we expect that any free albumin was separated from our sample. However, the drying of the TEM grids and then the vacuum conditions seem to have triggered the leaking of a part of the HSA coating and the partial formation of an HSA matrix on the TEM grid/SEM substrate.
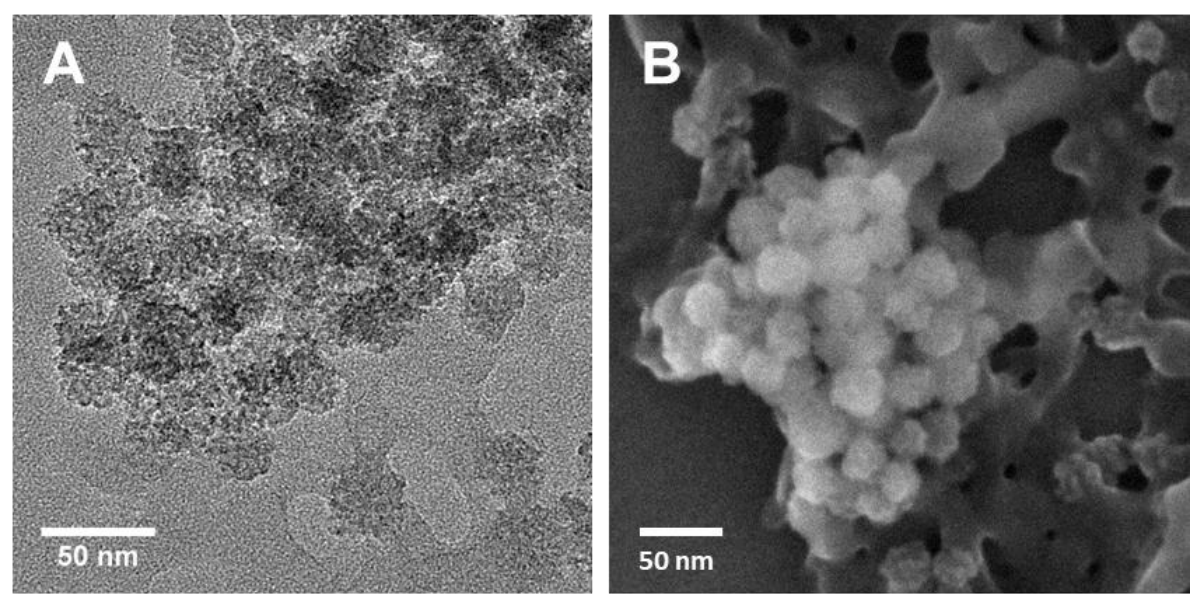

Figure 2. (A) TEM and (B) SEM images of RB-Dox-HSA-NPs in dried conditions from low to high magnifications respectively from the left to the right.

To ascertain this behaviour, AFM imaging in liquid phase was achieved on RB-DOX-HSA NPs in water (Figure 3). Careful inspection of images revealed aggregated and isolated particles uniformly distributed over the PEI-coated silicon wafer. Isolated nanoparticles were characterized by pseudo-spherical shape with a diameter in the range of $30-150 \mathrm{~nm}$. The average thickness of the isolated nanoparticles is about 
$67 \pm 23 \mathrm{~nm}$. Concerning the aggregated nanoparticles, they are randomly distributed over the silicon wafer surface and their size never exceeds 300-400 $\mathrm{nm}$ for a thickness of about 60-150 $\mathrm{nm}$. Moreover, the mappings of adhesion, energy dissipation and stiffness indicate homogeneity of both isolated and aggregated nanoparticles over the sample with average values of $285 \pm 49 \mathrm{pN}, 0.18 \pm 0.09 \mathrm{keV}$ and $2.28 \pm 0.17 \mathrm{MPa}$, respectively. The latter value is very low as compared to values previously reported for mesoporous silica.[38] Indeed, Young modulus of mesoporous silica is rather around 4-5 GPa and in the range of 10-100 MPa for nanocomposite gels blended with silica nanoparticles.[39,40] In our analysis, we found ca. $2 \mathrm{MPa}$ and this low value is attributed to the layer of HSA proteins that embedded the hard silica nanoparticles.

Regarding the drug payload, as some Dox was released during the IBAM and HSA steps, the main difficulty in such drug loading studies was to determine the final DLC in the RB-Dox-HSA NPs. Indeed, the common analysis of supernatants by fluorescence or absorbance spectroscopy may induce some errors taking into account the different synthesis steps. Therefore, TGA analyses were run on RB-Dox-HSA and RB-HSA NPs samples to evaluate the Dox loading by comparing the weight loss of both samples. Figure 4 shows the resulting TGA curves. The difference in weight loss between both samples suggested an efficient Dox loading. The first TGA curve on RB-HSA NPs indicated 53.3 wt.\% of RB and 46.7 wt. \% of HSA which corresponds to a protein coating content (CC) of $87.6 \%$. The second TGA curve obtained on RBDox-HSA NPs indicated 37.8 wt.\% of RB and 62.2 wt.\% of Dox-HSA. Considering the hypothesis that protein coating occurred in a similar way for both samples (with and without Dox) a CC of $87.6 \%$ would give a weight-percentage of $\sim 33 \mathrm{wt} . \%$ of HSA. Thus, the DLC of Dox is estimated at $\sim 29$ wt.\% in the RB nanosilica.
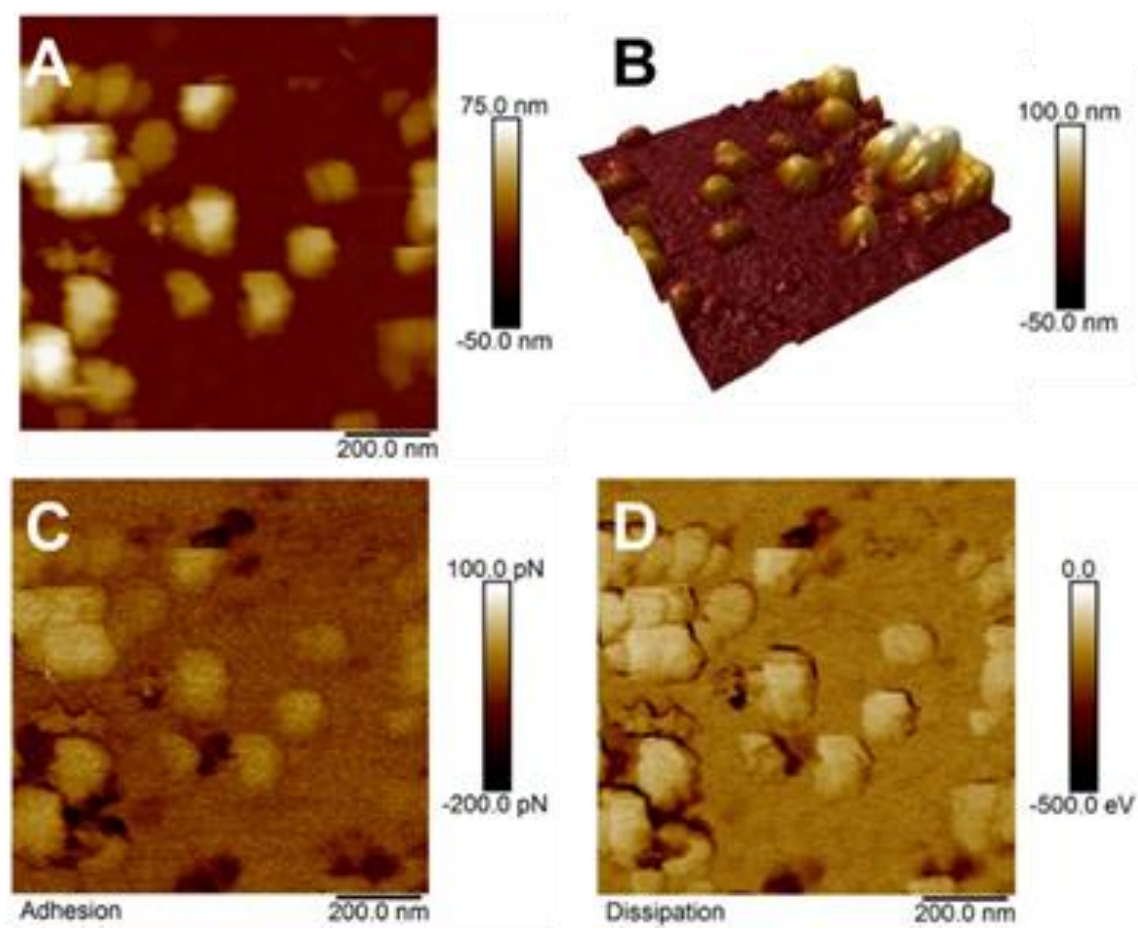

Figure 3. AFM images of RB-Dox-HSA-NPs in liquid conditions (water). 


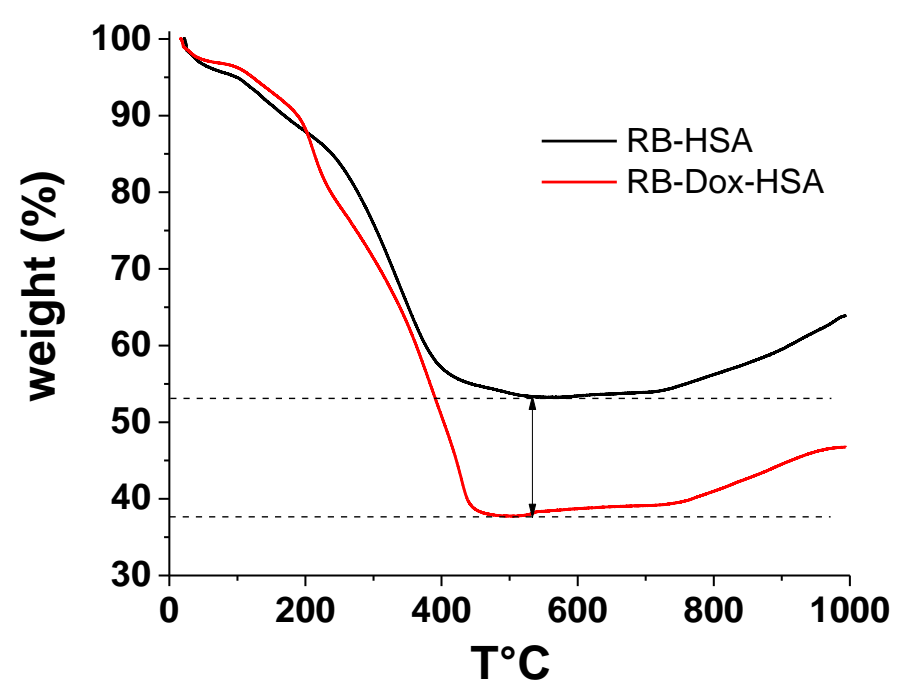

Figure 4. TGA curves of RB-Dox-HSA and RB-HSA samples.

\section{Silica removal and formation of DOX-HSA NPs}

With the aim to form silica free self-supported protein nanoparticles or capsules, RB sacrificial template was dissolved in $\mathrm{HF} / \mathrm{NH}_{4} \mathrm{~F}$ buffer. The resulting sample, Dox-HSA, was re-dispersed in water and formed a stable colloidal red suspension as pictured in Figure 5.A (photograph of the sample). Dynamic light scattering analysis (DLS) (intensity) performed on this sample, gave an average hydrodynamic size of $222 \pm 76 \mathrm{~nm}$ (Figure 5. B) indicating some effect of capsule aggregation which nevertheless did not lead to sedimentation with time.

Considering the DLC to be the percentage of the ratio between the mass of Dox and the mass of HSA capsules after complete dissolution of silica template, a high DLC value of $88 \%$ was estimated. This value was calculated from the previous TGA analysis on RB-Dox-HSA sample considering that the entire RB sacrificial template was dissolved. Table 1 compares DLC and DLE values of Dox loaded in HSA NPs reported in the literature[14,41-45] and the values obtained for our sample. Thus, compared to the various reported Dox encapsulation methods into HSA NPs, such as adsorption of Dox to preformed HSA NPs, incorporation of Dox to HSA matrix before desolvation or Dox conjugation to HSA molecules, our method of Dox soaking into sacrificial silica template led to high DLC's which is highly suitable for biological studies. That confirmed again that the formation of an aminosilane condensed at very high silane concentration is an efficient strategy to load high drug amount.[33]

Table 1. DLC and DLE values of Dox loading into HSA NPs from our sample compared to literature values

\begin{tabular}{|c|c|c|c|c|}
\hline Ref. & Type of Dox loading & Size (nm) & DLC (\%) & DLE (\%) \\
\hline Our work & Soaking into sacrificial silica template & 43 & 88 & - \\
\hline Bae et al. ${ }^{39}$ & Adsorption to preformed HSA NPs & 200 & 4.8 & 94.5 \\
\hline Xu et al. ${ }^{40}$ & $\begin{array}{c}\text { Conjugation via disulfide bonds (via RPA) + } \\
\text { physical adsorption }\end{array}$ & 30 & 56.2 & - \\
\hline
\end{tabular}



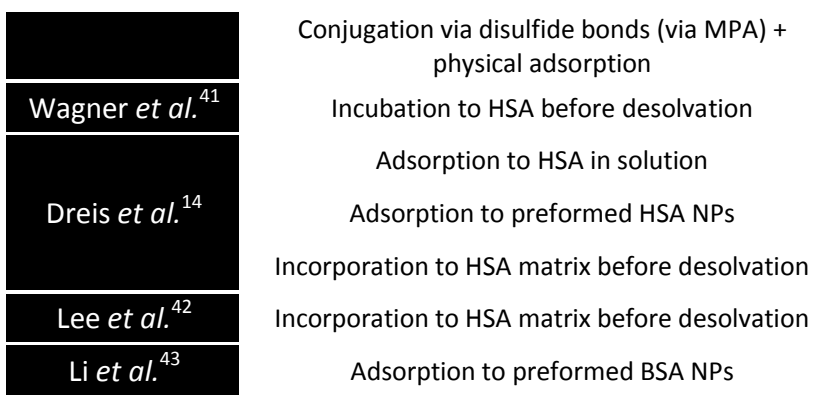

$\begin{array}{ccc} & 62.3 & - \\ 400 & 5.7 & - \\ - & 14 & 75 \\ 158 & 13.8 & 69 \\ 404 & 4.82 & 96.3 \\ 183 & 5 & - \\ 100 & 27.8 & 74\end{array}$

TEM analyses of the Dox-HSA sample were then performed. Three different kinds of morphologies were observed which may be due as described above to the TEM grids preparation and vacuum observation conditions. The first morphology, showed in Figure 5.C, was the major morphology observed for the Dox-HSA sample. This morphology was composed of a HSA network forming some kind of sticky like filaments that connected denser spherical HSA NPs together. These denser spherical HSA NPs exhibited sizes between 20 and $30 \mathrm{~nm}$ and thus, could correspond to HSA NPs formed from RB template dissolution as the RB NPs had sizes of $30 \mathrm{~nm}$. Previous hypotheses could explain the formation of this filamentous network as it was also observed on the TEM and SEM images of RB-Dox-HSA (grapes stuck into a HSA matrix in Figure 5). A first assumption is that the HSA coating through IBAM grafts was not tight enough and that HSA tend to desorb from the RB surface creating this kind of network. A second assumption could be that this network simply results from an effect of leaking through sample drying on TEM grid before imaging.

The second morphology, as shown in TEM images in Figure 5.D, exhibited spherical HSA NPs of $58 \pm$ $21 \mathrm{~nm}$. As this size was higher than the size of the original RB template, this could result for example from very thick HSA coatings on the RB NPs.

The third morphology, shown on TEM images in Figures 5.E, was typical for organic capsules. These capsules exhibited a size of $43 \pm 10 \mathrm{~nm}$ that corresponded to the RB sacrificial template size. This morphology should result from dried HSA NPs on the TEM grid.

Finally, the real morphology of the sample was rather difficult to determine through TEM and SEM analyses mostly because they involved drying this organic sample before imaging and imaging under vacuum conditions. 

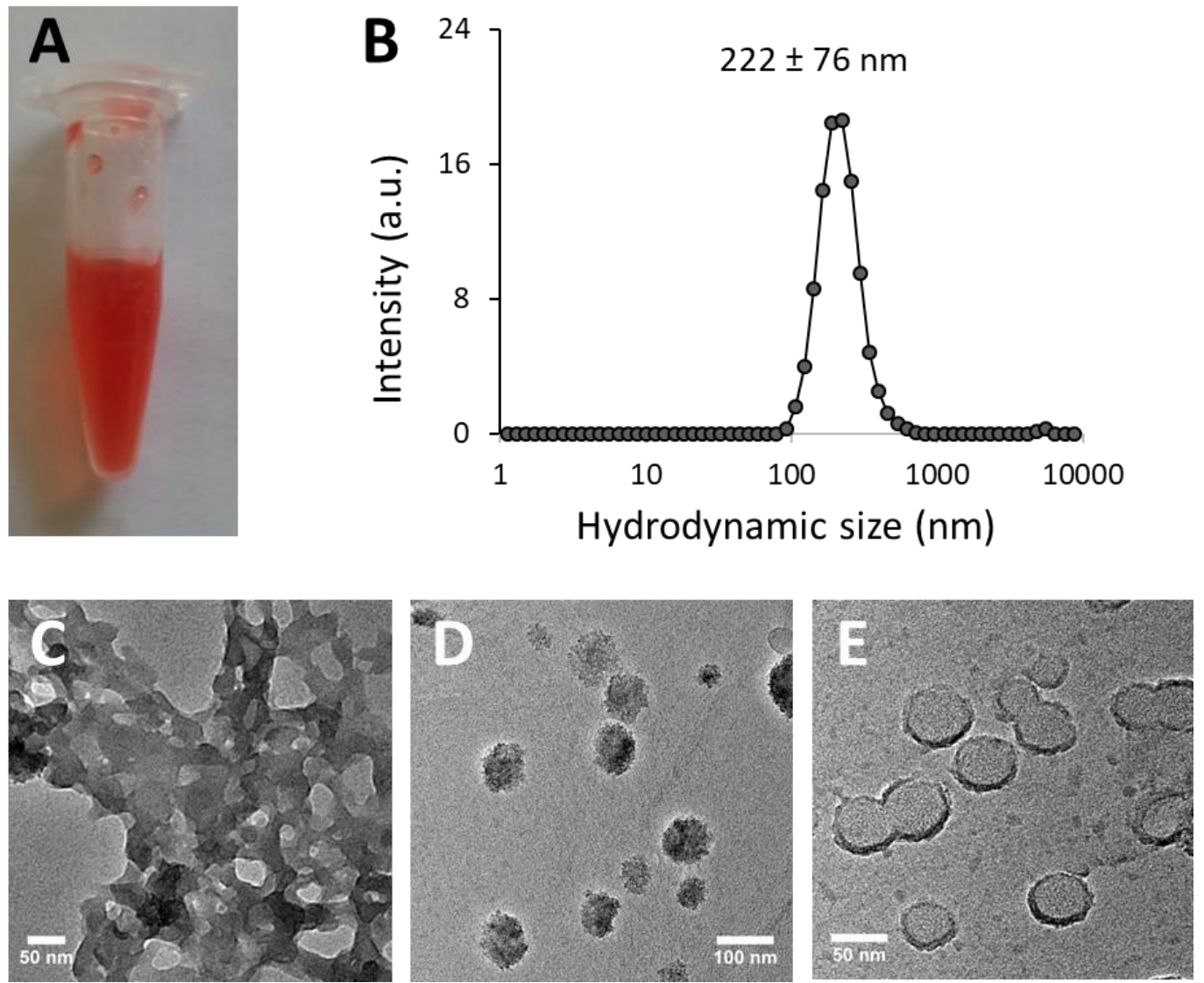

Figure 5.A photograph of the colloidal suspension of Dox-HSA NPs. B. DLS size distribution (intensity) of the red suspension. C-E. TEM images of the Dox-HSA NPs obtained after silica removal which showed three types of morphology : 1) HSA network like (C), 2) nanosphere like (D) and 3) nanocapsule like(E).

Thus to get additional insights in the morphology of the capsules in the hydrated state, we performed AFM in liquid conditions. AFM observations indicated that the HSA network (first morphology) was not anymore observed which tend to validate our previous hypothesis. AFM images showed that the Dox-HSA NPs in liquid conditions mostly appear as grapes of aggregated albumin nanospheres (Figure 6). The analysis of isolated nanoparticles evidenced pseudo-spherical shape with a diameter in the range of 50$150 \mathrm{~nm}$. The average thickness of the isolated nanoparticles was found about 30-70 nm. Concerning the aggregated nanoparticles, they were randomly distributed over the silicon wafer surface and their size were measured in the range of 250-1000 nm for a thickness of about 150-250 nm. Moreover, the mappings of adhesion, energy dissipation and stiffness indicated homogeneity of both isolated and aggregated nanoparticles over the sample with average value of $0.12 \pm 0.05 \mathrm{MPa}$. The latter value is consistent with the silica dissolution and its absence within the nanocapsules as compared with the value of $2 \mathrm{MPa}$ found in the presence of silica. Indeed, values between 0.01 to $0.5 \mathrm{MPa}$ were already reported for polyelectrolytes and hydrogels nanocapsules.[46-48] 

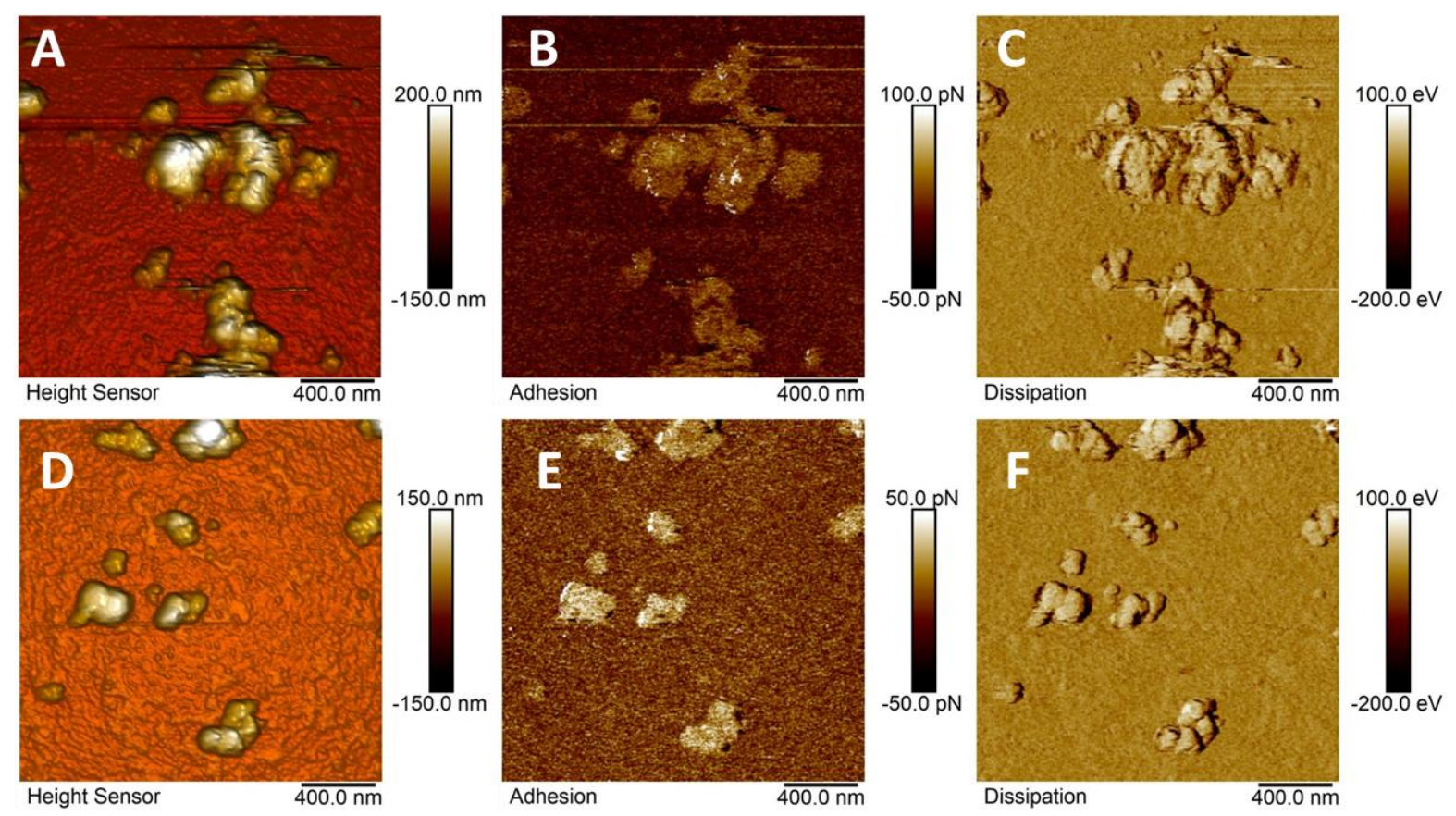

Figure 6. AFM imaging in liquid phase of the DOX-HSA capsules after silica removal forming aggregates of small capsules/vesicles mapped in two different zones (A and D). Images $(B, C)$ on one hand and $(E, F)$ on the other hand correspond respectively to adhesion and dissipation mapping associated respectively to the topographic images A and B.

\section{Drug delivery to multicellular spheroids and growth inhibition}

Dox-HSA capsules were assessed directly to cancer cells assuming that the diversity of the cell environment would provide triggers for Dox delivery like proteolysis by secreted enzyme or lysosomal enzyme. For that, cytotoxicity assays were performed on a Huh7 multicellular tumor spheroid model (Huh7 MCTS). Such model reproduces more closely the in vivo behavior of tumor cells than 2D culture system. Indeed, even if two-dimensional (2D) cell monolayers have been conventionally used by the scientific community to evaluate drug efficacy, NPs cytotoxicity and their internalization, they poorly represent the complex tumor 3D microenvironment. Indeed, cells grown in 2D lack the extracellular matrix (ECM), as well as cell-cell contact and cell-matrix interactions. Thus, 3D culture models have emerged as an additional in vitro step study to assist drug product development and tend to reduce the requirement for unnecessary animal studies [49-57]. Among various methods to engineer 3D cancer model such as spheroids, bio-printing and assemblies, we developed multicellular tumor spheroids (MCTS) cell culture by aggregation of cells in hanging drop which is one of the easiest ways to form 3D tumor model from cancer cell lines.

Thus we evaluated first the toxicity of Dox-HSA NPs and corresponding (Dox free) HSA NPs, by imaging the growth of the MCTS at various timepoints (2, 4 and 7 days). Huh7 MCTS were incubated with Dox-HSA and HSA NPs diluted in complete medium with concentrations of $35.4 \mu \mathrm{g} \cdot \mathrm{mL}^{-1}$ of HSA for both samples and a Dox concentration of $31 \mu \mathrm{g} \cdot \mathrm{mL}^{-1}$ for the Dox-HSA NPs sample. Figure 7 shows representative optical microscopy pictures of Huh7 MCTS at different days and with both NPs in comparison to control experiment (Mock). It shows that the HSA NPs did not modify the growth compared to non-treated MCTS 
however the cell growth for Dox-HSA NPs MCTS exhibited a strong inhibition and a different morphology that could be related to a massive cell death. Such images are characteristics of the Dox effect on MCTS.[58,59]

In the second series of experiments, measurements of MCTS surface area were also done after incubation of Dox-HSA NPs (17.7 $\mu \mathrm{g} \cdot \mathrm{mL}^{-1}$ of HSA and $15.5 \mu \mathrm{g} \cdot \mathrm{mL}^{-1}$ Dox $)$ and of HSA NPs $\left(17,7 \mu \mathrm{g} \cdot \mathrm{mL}^{-1}\right)$. The growth percentage was also calculated compared to the Mock. As shown on the results presented in Figure 8, Huh7 MCTS growth in presence of Dox-HSA NPs was readily inhibited. At this concentration tested, we can observe a decrease of $80 \%$ compared to the control. As the growth is not modified when Huh7 MCTS are incubated with HSA NPs, the effect may be related to Dox release. Our particles are made of human serum albumin and can be taken up by cells at least those of the outer layer of the cell aggregate by non-specific endocytosis and or pinocytosis. Dox could therefore be released after HSA degradation by lysosomal proteases. Furthermore, we expect from other experiments (not published yet) that Dox can diffuse through the entire Huh7 MCTS even if the NPs internalization occurred only at the spheroid borders. So the effect recorded here is a proof that Dox can be released from the nanocapsules.

$\begin{array}{lll}\text { D0 } & \text { D4 } & \text { D6 }\end{array}$

\section{C) Dox-HSA NPs}

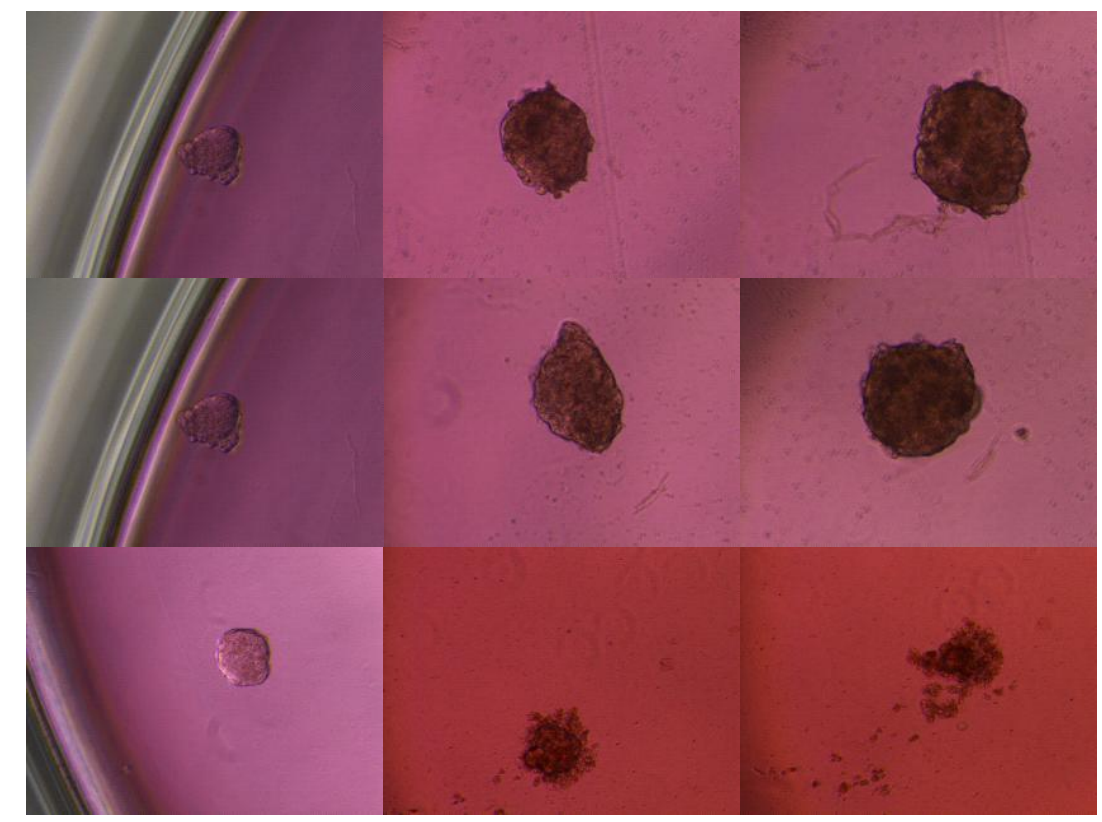

Figure 7. Photographs, taken by optical microscope (10X magnification), of A) non treated spheroids or Mock, B) spheroids treated with HSA NPs at $35.4 \mu \mathrm{g} \cdot \mathrm{mL}^{-1}$ of HSA and C) spheroids treated with Dox-HSA NPs at $35.4 \mu \mathrm{g} \cdot \mathrm{mL}^{-1}$ of HSA and $31 \mu \mathrm{g} \cdot \mathrm{mL}^{-1}$ of Doxorubicin at 0,4 and 6 days of incubation (D0, D4 and D6). 

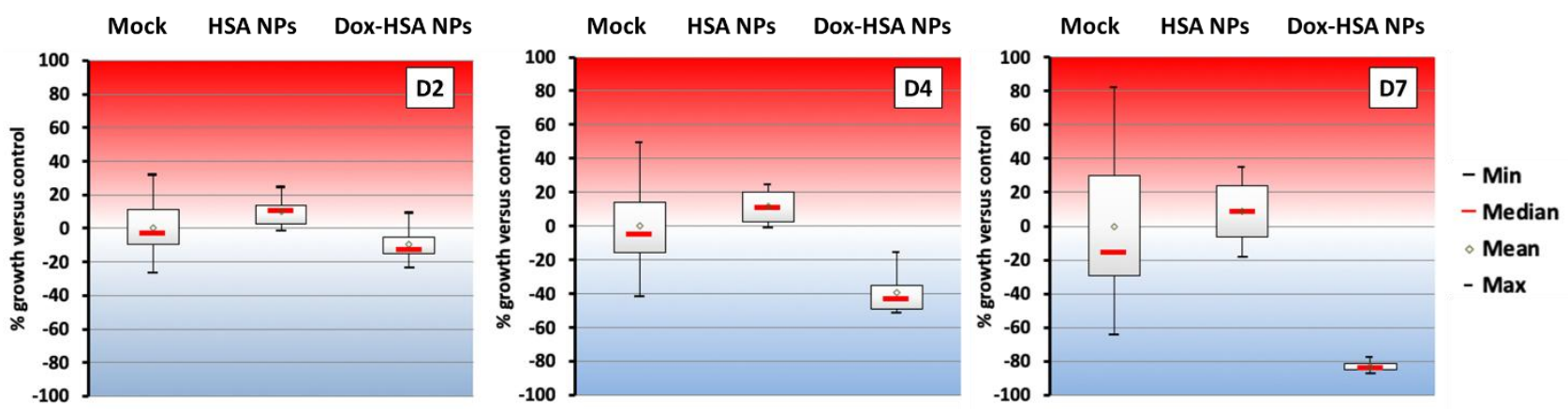

Figure 8. Box plot representing percentage of growth of Huh7 MCTS either treated with HSA NPs or DoxHSA NPs in comparison with non-treated ones (Mock) after 2, 4 and 7 days (D2, D4, D7) of incubation at concentrations of 17,7 $\mu \mathrm{g} \cdot \mathrm{mL}^{-1}$ of HSA and 15,5 $\mu \mathrm{g} \cdot \mathrm{mL}^{-1}$ Dox.

\section{Conclusion}

In this study, we reported the synthesis of small HSA nanocapsules loaded with doxorubicin (Dox) using mesoporous silica RB NPs as sacrificial templates. This sacrificial template based synthesis allowed hence i) HSA nanocapsules self-assembly without the use of toxic covalent cross-linkers , ii) nanometric sizes below $50 \mathrm{~nm}$ and iii) a high DLC of ca. 88\%. The efficacy of Dox-HSA nanocapsules was confirmed in multicellular tumor spheroid models (MCTS) of human hepatocarcinoma cells by their significant growth inhibition with respect to controls. In future works, modification of the protein surface of the capsules for cell targeting or to improve colloidal stability and low fouling properties are envisioned. As shown herein, our approach opens a new synthesis route in the field of protein-based nanocarriers for drug delivery applications.

\section{Conflicts of interest.}

There are no conflicts to declare.

\section{Acknowledgements.}

The French Ministry of Research is gratefully acknowledged for financial support of M.M. doctoral fellowship. This work was also supported from Alsace contre le cancer, VEnaCOCO project. D.M. acknowledges the IDEX-attractivité funding scheme from the University of Strasbourg. The Institut National du Cancer (INCa) is also thanked for financial support ((project PRTK14,THERAMAG 2014-225). We also thank Didier Burger for running the TGA analyses.

\section{Supporting information:}

S1. $\mathrm{N}_{2}$ adsorption-desorption isotherms; S2. FTIR spectra of RB and $\mathrm{RB}-\mathrm{NH}_{2}$. And S3. FTIR spectra of RB-Dox-HSA and RB-HSA samples compared to RB. 


\section{References}

[1] L. Rochette, C. Guenancia, A. Gudjoncik, O. Hachet, M. Zeller, Y. Cottin, C. Vergely, Anthracyclines/trastuzumab: new aspects of cardiotoxicity and molecular mechanisms, Trends Pharmacol. Sci., 36 (2015) 326-348.

[2] K. Ylänen, T. Poutanen, P. Savikurki-Heikkilä, I. Rinta-Kiikka, A. Eerola, K. Vettenranta, Cardiac Magnetic Resonance Imaging in the Evaluation of the Late Effects of Anthracyclines Among Long-Term Survivors of Childhood Cancer, J. Am. Coll. Cardiol., 61 (2013) 1539-1547.

[3] C. Elalouani, M.A. Benhmidoun, H. Rida, M. AitRaiss, N. Derhem, A. Elomrani, M. Khouchani, A. Tahri, A. Errehmouni, R. Faouzi, A. Elguenzri, M. Elhattaoui, I. Tazi, L. Mahmal, Cardiotoxicité à court et à moyen terme des anthracyclines : étude prospective, Ann. Cardiol. Angéiologie, 61 (2012) 257-266.

[4] M. Cruz, J. Duarte-Rodrigues, M. Campelo, Cardiotoxicity in anthracycline therapy: Prevention strategies, Rev. Port. Cardiol. Engl. Ed., 35 (2016) 359-371.

[5] Á. Ancochea, A. Salar, F. García-Pallarols, E. Gimeno, C. Fernández-Rodriguez, B. Sánchez-González, Prognostic impact of anthracyclines in the treatment of aggressive lymphoma in patients over 70 years, Med. Clínica Engl. Ed., 144 (2015) 544-547.

[6] F. Kratz, Albumin as a drug carrier: Design of prodrugs, drug conjugates and nanoparticles, J. Controlled Release, 132 (2008) 171-183.

[7] B. Elsadek, F. Kratz, Impact of albumin on drug delivery - New applications on the horizon, J. Controlled Release, 157 (2012) 4-28.

[8] K. Langer, M.G. Anhorn, I. Steinhauser, S. Dreis, D. Celebi, N. Schrickel, S. Faust, V. Vogel, Human serum albumin (HSA) nanoparticles: Reproducibility of preparation process and kinetics of enzymatic degradation, Int. J. Pharm., 347 (2008) 109-117.

[9] Y. Matsumura, H. Maeda, A New Concept for Macromolecular Therapeutics in Cancer Chemotherapy: Mechanism of Tumoritropic Accumulation of Proteins and the Antitumor Agent Smancs, Cancer Res., 46 (1986) 6387-6392.

[10] C. Weber, C. Coester, J. Kreuter, K. Langer, Desolvation process and surface characterisation of protein nanoparticles, Int. J. Pharm., 194 (2000) 91-102.

[11] K. Langer, S. Balthasar, V. Vogel, N. Dinauer, H. von Briesen, D. Schubert, Optimization of the preparation process for human serum albumin (HSA) nanoparticles, Int. J. Pharm., 257 (2003) 169-180.

[12] H. Wartlick, B. Spänkuch-Schmitt, K. Strebhardt, J. Kreuter, K. Langer, Tumour cell delivery of antisense oligonuclceotides by human serum albumin nanoparticles, J. Controlled Release, 96 (2004) 483-495.

[13] A.O. Elzoghby, W.M. Samy, N.A. Elgindy, Albumin-based nanoparticles as potential controlled release drug delivery systems, J. Controlled Release, 157 (2012) 168-182.

[14] S. Dreis, F. Rothweiler, M. Michaelis, J. Cinatl Jr., J. Kreuter, K. Langer, Preparation, characterisation and maintenance of drug efficacy of doxorubicin-loaded human serum albumin (HSA) nanoparticles, Int. J. Pharm., 341 (2007) 207-214.

[15] E. Zeiger, B. Gollapudi, P. Spencer, Genetic toxicity and carcinogenicity studies of glutaraldehyde - a review, Mutat. Res. Mutat. Res., 589 (2005) 136-151.

[16] W. Fürst, A. Banerjee, Release of Glutaraldehyde From an Albumin-Glutaraldehyde Tissue Adhesive Causes Significant In Vitro and In Vivo Toxicity, Ann. Thorac. Surg., 79 (2005) 1522-1528.

[17] G. Speit, S. Neuss, P. Schütz, M. Fröhler-Keller, O. Schmid, The genotoxic potential of glutaraldehyde in mammalian cells in vitro in comparison with formaldehyde, Mutat. Res. Toxicol. Environ. Mutagen., 649 (2008) 146-154. 
[18] R.A. Petros, J.M. DeSimone, Strategies in the design of nanoparticles for therapeutic applications, Nat. Rev. Drug Discov., 9 (2010) 615.

[19] S. Mitragotri, J. Lahann, Physical approaches to biomaterial design, Nat. Mater., 8 (2009) 15.

[20] D. Mertz, P. Tan, Y. Wang, T.K. Goh, A. Blencowe, F. Caruso, Bromoisobutyramide as an Intermolecular Surface Binder for the Preparation of Free-standing Biopolymer Assemblies, Adv. Mater., 23 (2011) 5668-5673.

[21] D. Mertz, J. Cui, Y. Yan, G. Devlin, C. Chaubaroux, A. Dochter, R. Alles, P. Lavalle, J.C. Voegel, A. Blencowe, P. Auffinger, F. Caruso, Protein Capsules Assembled via Isobutyramide Grafts: Sequential Growth, Biofunctionalization, and Cellular Uptake, ACS Nano, 6 (2012) 7584-7594.

[22] D. Mertz, C. Affolter-Zbaraszczuk, J. Barthès, J. Cui, F. Caruso, T.F. Baumert, J.-C. Voegel, J. Ogier, F. Meyer, Templated assembly of albumin-based nanoparticles for simultaneous gene silencing and magnetic resonance imaging, Nanoscale, 6 (2014) 11676-11680.

[23] P. Yang, S. Gai, J. Lin, Functionalized mesoporous silica materials for controlled drug delivery, Chem. Soc. Rev., 41 (2012) 3679-3698.

[24] F. Tang, L. Li, D. Chen, Mesoporous silica nanoparticles: synthesis, biocompatibility and drug delivery, Adv. Mater., 24 (2012) 1504-1534.

[25] G.J. de A. Soler-Illia, C. Sanchez, B. Lebeau, J. Patarin, Chemical strategies to design textured materials: from microporous and mesoporous oxides to nanonetworks and hierarchical structures, Chem. Rev., 102 (2002) 4093-4138.

[26] J. Cui, M.P. van Koeverden, M. Müllner, K. Kempe, F. Caruso, Emerging methods for the fabrication of polymer capsules, Adv. Colloid Interface Sci., 207 (2014) 14-31.

[27] M. Björnmalm, J. Cui, N. Bertleff-Zieschang, D. Song, M. Faria, M.A. Rahim, F. Caruso, Nanoengineering Particles through Template Assembly, Chem. Mater., 29 (2016) 289-306.

[28] M. Vallet-Regi, A. Rámila, R.P. del Real, J. Pérez-Pariente, A New Property of MCM41: Drug Delivery System, Chem. Mater., 13 (2001) 308-311.

[29] N.Ž. Knežević, J.-O. Durand, Large pore mesoporous silica nanomaterials for application in delivery of biomolecules, Nanoscale, 7 (2015) 2199-2209.

[30] M. Vallet-Regí, F. Balas, D. Arcos, Mesoporous Materials for Drug Delivery, Angew. Chem. Int. Ed., 46 (2007) 7548-7558.

[31] S. Wang, Ordered mesoporous materials for drug delivery, Microporous Mesoporous Mater., 117 (2009) 1-9.

[32] K. Zhang, L.-L. Xu, J.-G. Jiang, N. Calin, K.-F. Lam, S.-J. Zhang, H.-H. Wu, G.-D. Wu, B. Albela, L. Bonneviot, P. Wu, Facile Large-Scale Synthesis of Monodisperse Mesoporous Silica Nanospheres with Tunable Pore Structure, J. Am. Chem. Soc., 135 (2013) 2427-2430.

[33] C. Wells, O. Vollin- Bringel, V. Fiegel, S. Harlepp, B.V. der Schueren, S. Bégin- Colin, D. Bégin, D. Mertz, Engineering of Mesoporous Silica Coated Carbon-Based Materials Optimized for an Ultrahigh Doxorubicin Payload and a Drug Release Activated by $\mathrm{pH}$, T, and NIR-light, Adv. Funct. Mater., 28 (n.d.) 1706996.

[34] N.E. Timmins, L.K. Nielsen, Generation of multicellular tumor spheroids by the hanging-drop method, Tissue Eng., (2007) 141-151.

[35] I.N. Sneddon, The relation between load and penetration in the axisymmetric Boussinesq problem for a punch of arbitrary profile, Int. J. Eng. Sci., 3 (1965) 47-57.

[36] N. Gavara, R.S. Chadwick, Determination of the elastic moduli of thin samples and adherent cells using conical atomic force microscope tips, Nat. Nanotechnol., 7 (2012) 733. 
[37] P. Polyakov, C. Soussen, J. Duan, J.F. Duval, D. Brie, G. Francius, Automated force volume image processing for biological samples, PLoS One, 6 (2011) e18887.

[38] S. Dourdain, D.T. Britton, H. Reichert, A. Gibaud, Determination of the elastic modulus of mesoporous silica thin films by $\mathrm{x}$-ray reflectivity via the capillary condensation of water, Appl. Phys. Lett., 93 (2008) 183108.

[39] M.S. Islam, R. Masoodi, H. Rostami, The Effect of Nanoparticles Percentage on Mechanical Behavior of Silica-Epoxy Nanocomposites, J. Nanosci., (2013).

[40] J. Liu, G. Zong, L. He, Y. Zhang, C. Liu, L. Wang, Effects of fumed and mesoporous silica nanoparticles on the properties of Sylgard 184 polydimethylsiloxane, Micromachines, 6 (2015) 855-864.

[41] S. Bae, K. Ma, T.H. Kim, E.S. Lee, K.T. Oh, E.-S. Park, K.C. Lee, Y.S. Youn, Doxorubicin-loaded human serum albumin nanoparticles surface-modified with TNFrelated apoptosis-inducing ligand and transferrin for targeting multiple tumor types, Biomaterials, 33 (2012) 1536-1546.

[42] R. Xu, M. Fisher, R.L. Juliano, Targeted Albumin-Based Nanoparticles for Delivery of Amphipathic Drugs, Bioconjug. Chem., 22 (2011) 870-878.

[43] S. Wagner, F. Rothweiler, M.G. Anhorn, D. Sauer, I. Riemann, E.C. Weiss, A. KatsenGloba, M. Michaelis, J. Cinatl Jr., D. Schwartz, J. Kreuter, H. von Briesen, K. Langer, Enhanced drug targeting by attachment of an anti $\alpha \mathrm{v}$ integrin antibody to doxorubicin loaded human serum albumin nanoparticles, Biomaterials, 31 (2010) 2388-2398.

[44] H. Lee, S. Park, J.B. Kim, J. Kim, H. Kim, Entrapped doxorubicin nanoparticles for the treatment of metastatic anoikis-resistant cancer cells, Cancer Lett., 332 (2013) 110-119.

[45] F. Li, C. Zheng, J. Xin, F. Chen, H. Ling, L. Sun, T.J. Webster, X. Ming, J. Liu, Enhanced tumor delivery and antitumor response of doxorubicin loaded albumin nanoparticles formulated based on a Schiff base, Int. J. Nanomedicine, (2016).

[46] M.V. Flores-Merino, S. Chirasatitsin, C. LoPresti, G.C. Reilly, G. Battaglia, A.J. Engler, Nanoscopic mechanical anisotropy in hydrogel surfaces, Soft Matter, 6 (2010) 44664470.

[47] J. Zaragoza, A. Chang, P. Asuri, Effect of crosslinker length on the elastic and compression modulus of poly (acrylamide) nanocomposite hydrogels, in: J. Phys. Conf. Ser., IOP Publishing, 2017: p. 012037.

[48] L.A. Frank, G. Sandri, F. D’Autilia, R.V. Contri, M.C. Bonferoni, C. Caramella, A.G. Frank, A.R. Pohlmann, S.S. Guterres, Chitosan gel containing polymeric nanocapsules: a new formulation for vaginal drug delivery, Int. J. Nanomedicine, 9 (2014) 3151.

[49] G. Hamilton, Multicellular spheroids as an in vitro tumor model, Cancer Lett., 131 (1998) 29-34.

[50] L.A. Kunz- Schughart, Multicellular tumor spheroids: intermediates between monolayer culture and in vivo tumor, Cell Biol. Int., 23 (1999) 157-161.

[51] R.-Z. Lin, H.-Y. Chang, Recent advances in three-dimensional multicellular spheroid culture for biomedical research, Biotechnol. J., 3 (2008) 1172-1184.

[52] F. Hirschhaeuser, H. Menne, C. Dittfeld, J. West, W. Mueller-Klieser, L.A. KunzSchughart, Multicellular tumor spheroids: An underestimated tool is catching up again, J. Biotechnol., 148 (2010) 3-15.

[53] D.W. Hutmacher, Biomaterials offer cancer research the third dimension, Nat. Mater., 9 (2010) 90-93.

[54] G. Mehta, A.Y. Hsiao, M. Ingram, G.D. Luker, S. Takayama, Opportunities and challenges for use of tumor spheroids as models to test drug delivery and efficacy, J. Controlled Release, 164 (2012) 192-204. 
[55] K.A. Fitzgerald, M. Malhotra, C.M. Curtin, F.J. O’ Brien, C.M. O' Driscoll, Life in 3D is never flat: 3D models to optimise drug delivery, J. Controlled Release, 215 (2015) 3954.

[56] W. Asghar, R. El Assal, H. Shafiee, S. Pitteri, R. Paulmurugan, U. Demirci, Engineering cancer microenvironments for in vitro 3-D tumor models, Mater. Today, 18 (2015) 539553.

[57] A.K. Mapanao, M. Santi, P. Faraci, V. Cappello, D. Cassano, V. Voliani, Endogenously Triggerable Ultrasmall-in-Nano Architectures: Targeting Assessment on 3D Pancreatic Carcinoma Spheroids, ACS Omega, 3 (2018) 11796-11801.

[58] X. Wang, X. Zhen, J. Wang, J. Zhang, W. Wu, X. Jiang, Doxorubicin delivery to 3D multicellular spheroids and tumors based on boronic acid-rich chitosan nanoparticles, Biomaterials, 34 (2013) 4667-4679.

[59] S. Eetezadi, R. De Souza, M. Vythilingam, R. Lessa Cataldi, C. Allen, Effects of Doxorubicin Delivery Systems and Mild Hyperthermia on Tissue Penetration in 3D Cell Culture Models of Ovarian Cancer Residual Disease, Mol. Pharm., 12 (2015) 39733985. 


\section{SUPPORTING INFORMATION}

\section{Mesoporous silica templated-albumin nanoparticles with high doxorubicin payload for drug delivery assessed with a 3-D tumor cell model}

Mathilde Ménarda,b, Florent Meyer*,b, Ksenia Parkhomenkoc ${ }^{c}$ Cédric Leuvreya ${ }^{a}$ Grégory Francius ${ }^{\mathrm{d}}$, Sylvie Bégin-Colina, Damien Mertz ${ }^{*}$,a

a Institut de Physique et Chimie des Matériaux de Strasbourg (IPCMS), UMR 7504, CNRS, Université de Strasbourg, 23, rue du Loess, BP 43, 67034 Strasbourg, France. E-mails: damien.mertz@ipcms.unistra.fr sylvie.begin@ipcms.unistra.fr

b Université de Strasbourg, INSERM, UMR_S 1121 Biomatériaux et bioingénierie, FMTS, 11 rue Humann, 67085 Strasbourg, Cedex, France. E-mail : fmeyer@unistra.fr

'Institut de Chimie et Procédés pour l'Energie l'Environnement et la Santé, 25 rue Becquerel 67087 Strasbourg

${ }^{d}$ CNRS - Université de Lorraine, Laboratoire de Chimie Physique et Microbiologie pour l'Environnement, LCPME, UMR 7564, Villers-lès-Nancy, F-54600, France 

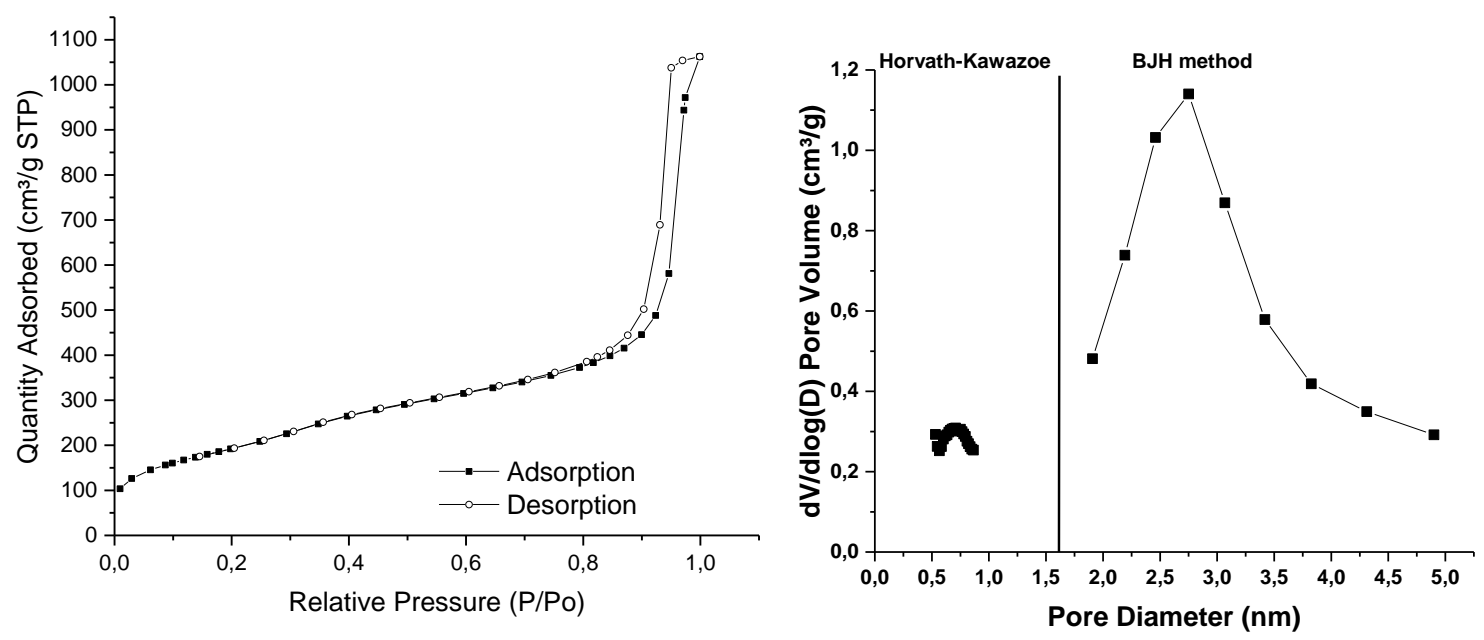

Figure S1. A) $\mathrm{N}_{2}$ adsorption-desorption isotherms of RB NPs and B) their corresponding pore size distribution curves in inset calculated by the BJH method for pore sizes above $2 \mathrm{~nm}$ and the HorvathKawazoe method for pore sizes $<1 \mathrm{~nm}$. 


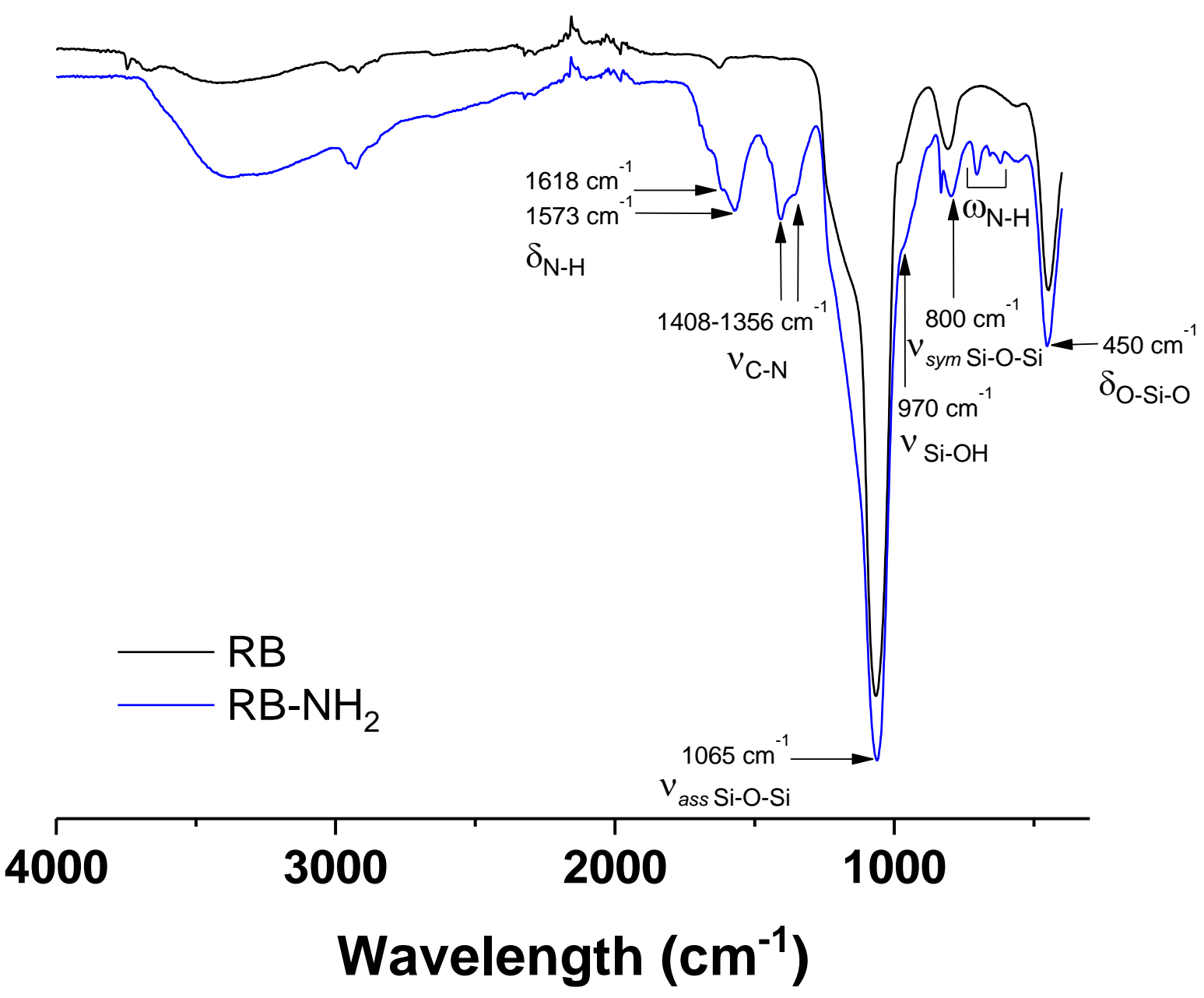

Figure S2. FTIR spectra of RB and RB- $\mathrm{NH}_{2}$. 


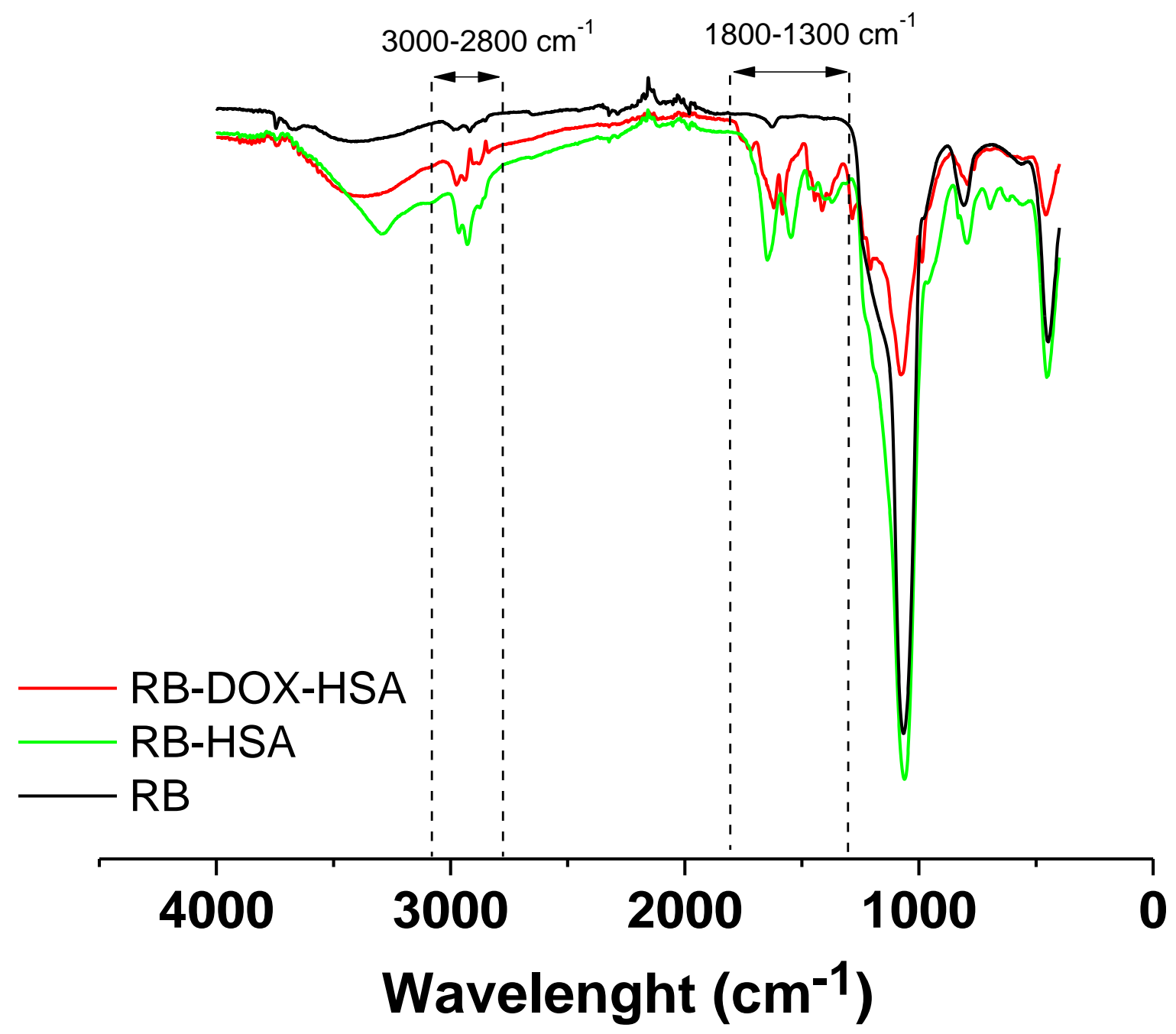

Figure S3. (A) ATR-IR analysis of RB-Dox-HSA and RB-HSA samples compared to RB. 